\title{
Acute Urinary Retention: Should We Call It a Manifestation of Appendicitis?
}

\author{
(1) Sadaf Sheikh, (D) Umair Javed, (1) Muhammad Akbar Baig \\ Department of Emergency Medicine, Aga Khan University Hospital, Karachi, Pakistan
}

\begin{abstract}
Acute urinary retention is a fairly common presentation in the elderly but it is a rare manifestation of acute appendicitis in a healthy young individual. We presented a 32-year-old male with decreased urine output with mild tenderness at the suprapubic region later on shifted to right iliac fossa. Computed tomography of abdomen showed acute appendicitis. Emergency physicians should recognize the uncommon presentation of acute appendicitis and should be aware of this common complaint as a rare presentation. This case also highlights the importance of repeated abdominal examinations to seek diagnostic clarity.
\end{abstract}

Keywords: Appendicitis, acute urinary retention, repeat abdominal examination

\section{Introduction}

The classical presentation of acute appendicitis is well known: periumbilical pain radiating to right iliac fossa with nausea, vomiting and anorexia. Yet, this presentation is not always there to indicate the diagnosis. Less is been published regarding atypical presentation of acute appendicitis and one needs to think of alternative diagnosis in the setting of acute urinary retention (AUR).

\section{Case Report}

A previously healthy 32-year-old gentleman, presented to emergency room with complain of inability to pass urine for the past 8 hours associated with low abdominal pain. Lower abdominal pain was non-radiating, dull in character with no clear aggravation and relieving factor. There was no associated nausea and vomiting. He reported normal appetite and no history of fever. He had no prior urological history and denies any history of urethral discharge. Sexual history was unremarkable. Past surgical and medical history was unremarkable. Moreover, there was no prior history of kidney stones or urethral stricture. Bowel habits were normal and patient denied any addiction.

On arrival patient was afebrile, had heart rate of $76 \mathrm{bpm}$, blood pressure of $126 / 72 \mathrm{mmHg}$, respiratory rate of $18 / \mathrm{min}$ and oxygen saturation on pulse oximeter of $99 \%$ at room air. General physical examination was unremarkable except for dry mucosa and skin. Abdominal examination revealed soft abdomen with mild tenderness in lower abdomen. Bladder was palpable midway between symphysis pubis and umbilicus. Normal gut sounds were found on auscultation. There was no rebound tenderness or costo-vertebral tenderness. Rest of the systemic examination was unremarkable. Genitourinary examination was also unremarkable.

Foley catheterization was done without any complication. Post void residual of $400 \mathrm{~mL}$ was obtained. (Table 1).

Urinalysis reported negative leucocyte esterase, nitrites and leukocytes with hemoglobin 1+ and ketone 1+ on dipstick testing.

Cite this article as: Sheikh S, Javed U, Baig MA. Acute urinary retention: Should we call it a manifestation of appendicitis? Eurasian J Emerg Med. 2020;19(1):52-4. 
Reassessment performed after 2 hours revealed that lower abdominal pain did not settle. Abdominal examination after 2 hours revealed lower abdominal tenderness more marked in right iliac fossa. Rovsing sign, obturator sign and psoas sign were negative. Rebound tenderness was positive. Alvarado score was 5 points ( 1 for rebound tenderness, 1 for right iliac fossa tenderness, 2 for elevated leukocyte count and 1 for leukocyte left shift).

Focus assessment computed tomography reported dilated appendix of $10.5 \mathrm{~mm}$ with multiple high density foci within its lumen representing appendicoliths. Mild free fluid and periappendiceal inflammatory changes were present (Figure 1, 2).

\begin{tabular}{|l|l|}
\hline Table 1. Initial laboratory investigations were as follows \\
\hline Hemoglobin & 15.9 \\
\hline Hematocrit & 46.1 \\
\hline Mean corpuscular volume & 82.5 \\
\hline White blood cells & 14.1 \\
\hline Neutrophils & 88.1 \\
\hline Lymphocytes & 5.2 \\
\hline Platelets & 265 \\
\hline Blood urea nitrogen & 9 \\
\hline Creatinine & 1 \\
\hline Sodium & 135 \\
\hline Potassium & 3.3 \\
\hline Chloride & 96 \\
\hline Bicarbonate & 28.8 \\
\hline Urine cultures & - \\
\hline
\end{tabular}

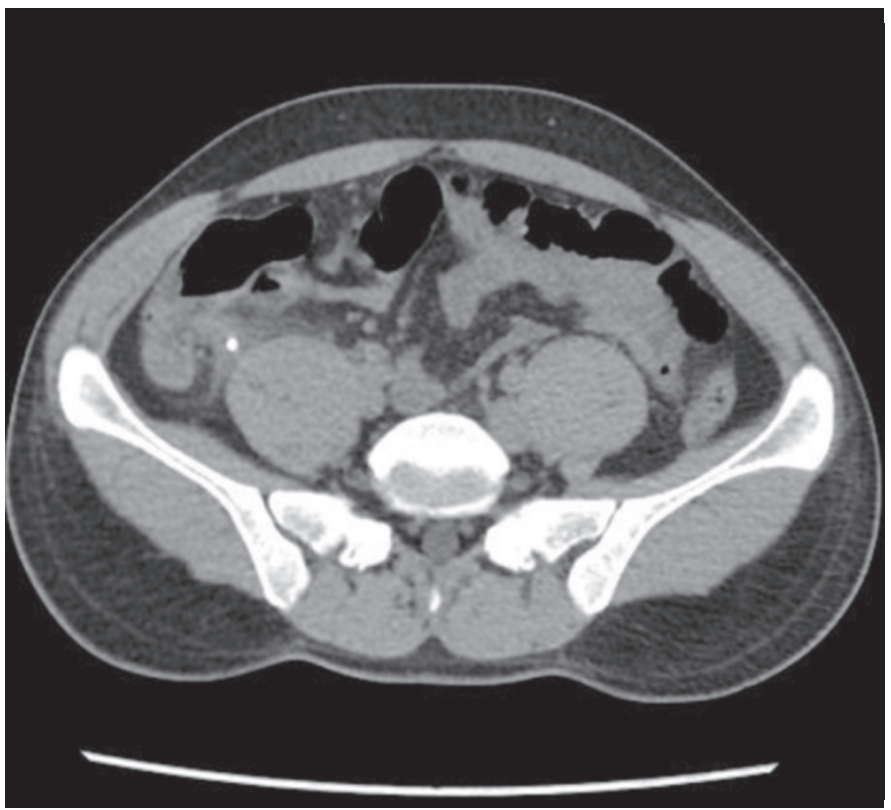

Figure 1. Image showing appendicolith

\section{Discussion}

Acute appendicitis is the high volume diagnosis in the emergency room. As emergency physicians, we don't always consider the mimicking conditions of

acute appendicitis, moreover it has a complex and atypical range of presentation leading to missed diagnosis (1).

It is common to operate an ureteric stone with a pre-operative diagnosis of appendicitis but on the contrary, mistakes could happen in the case of urological manifestations of acute appendicitis.

This case report focused on the variability in manifestation of acute appendicitis notably AUR and hematuria. This variability is likely originating from the location of appendix leading to diagnostic dilemmas. Appendicitis manifesting as AUR is rare. Urological manifestations of acute appendicitis which may otherwise point to the pathology of genito-urinary system, could be dysuria, urinary frequency, AUR, rectal pain or testicular pain (2).

Dysuria is the result of irritation of urinary bladder in case of peri-appendiceal inflammation. In case of pelvic appendix, it's not common to have abdominal rigidity misleading the examiner to think about bladder pathology. Patients complain that they get full bladder but have no complaints about passing urine (2). It is mainly when appendix is behind cecum, in the iliac fossa or deep in the pelvis.

Retention of urine is due to the irritation of bladder sphincter which is uncommon. Difficulty in urination is the common complaint in this situation (2).

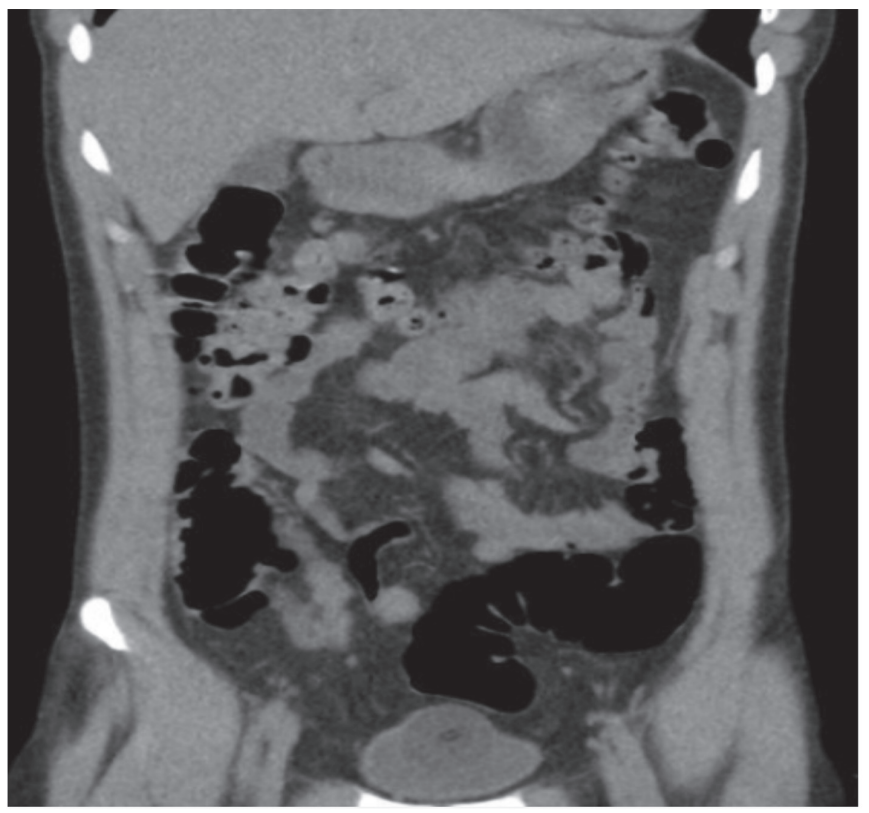

Figure 2. Image showing peri-appendiceal inflammation 
Differential diagnosis is relatively easier when urine is well examined. While differentiating it from pyelitis, acute appendicitis is associated with normal urine analysis with rigidity of abdominal muscles and fever. Tenderness at right erector-costal angle does present in the acute appendicitis due to irritation of tenth dorsal spinal nerve which innervates the appendix (2). Literature showed such rare presentation in acute appendicitis with gross hematuria, acute prostatitis and acute pyelonephritis $(3,4)$. Such unusual manifestations should be sought in the differential diagnosis of AUR.

\section{Ethics}

Informed Consent: Informed consent was obtained.

Peer-review: Externally peer-reviewed.

\section{Authorship Contributions}

Concept: S.S, U.J., M.A.B., Design: S.S, U.J., M.A.B., Data Collection or Processing: S.S, U.J., M.A.B., Analysis or Interpretation: S.S,
U.J., M.A.B., Literature Search: S.S, U.J., M.A.B., Writing: S.S, U.J., M.A.B.

Conflict of Interest: No conflict of interest was declared by the authors.

Financial Disclosure: The authors declared that this study received no financial support.

\section{References}

1. Munson RJ, Fontanarosa PB, Gerson L. Acute appendicitis in elderly: Clinical features of early and delayed diagnoses. Ann Emerg Med.1992;21:636-7.

2. V. Zachary Cope. Genito-Urinary Symptoms in Acute Appendicitis. First Published June 1, 1922 Meeting Report.

3. Fontanarosa PB, Roush WR. Acute urinary retention. Emerg Med Clin North Am.1988;6:419-37.

4. Jones WG, Barie PS. Urological manifestations of acute appendicitis. J Urol. 1988;139:1325-8 